\title{
Topographic Distribution of Carious Lesion on Young Permanent Mandibular Molars and Its Relation to Periapical Index Score of Apical Periodontitis: A Radiographic Analysis
}

INTRODUCTION: Caries in young permanent teeth progress rapidly resulting in early pulp involvement which further progresses into loss of mineralized tissue and severe periapical pathologies. The Periapical Index scoring system (PAI) proposed by Ørstavik et al. in 1986 is an invaluable diagnostic tool used to evaluate periapical lesion extent and severity by $2 \mathrm{D}$ digital intraoral periapical radiographic analysis with minimal radiation exposure and hence treatment planning.

AIM: To evaluate the relationship of the surface distribution of caries in young permanent molars, pulpal involvement and periapical index (PAI) score with respect to individual root forming young permanent mandibular molars.

MATERIALS AND METHOD: In this retrospective single-centre study, 100 pulpally involved carious young permanent mandibular molars were included, selected from the departmental records between August 2018-2019. Topographic distribution of caries and radiographic analysis (using PAI) was evaluated to see the effect of anatomical site of caries, individual anatomical form of root, and extent \& severity of apical periodontitis developed.

RESULT: No significant correlation between the site of caries involvement and PAI score was observed. PAI score for distal roots was higher(PAI> 2) as compared to mesial root for pulpally involved carious young permanent first molars (p-value mesial root-0.576, distal root-0.591) CONCLUSION: PAI score is independent of the topographic distribution of caries and no correlation was observed. Periapical radiolucency does not depend on caries distribution, either it is mesial, distal or occlusal.

KEYWORDS: Topography, Caries Distribution, Periapical Radiolucency, Periapical Index

\section{INTRODUCTION}

According to the World Health Organization, dental caries is the most prevalent disease globally and affects $60-90 \%$ of school children in most industrialized nations. ${ }^{1-3}$

Pulp infection mainly occurs as a consequence of dental caries, trauma, and restorative procedures and frequently proceeds to pulp necrosis and apical periodontitis with localized bone destruction. ${ }^{4}$ In these circumstances, the apical periodontium, formed by cementum, periodontal ligament, and alveolar bone in the root apex region, is the site where complex inflammatory reactions take place. ${ }^{4}$ The extent of the caries of a tooth is important for periapical health. Various cross-sectional studies have been done on the prevalence and frequencies of Apical Periodontitis (AP) and demonstrated that $30-50 \%$ of individuals exhibit AP. 5 The presence and radiographic quality of a root filling and to some extent the radiographic quality of the coronal restoration was associated with apical periodontitis in teeth. ${ }^{6}$ The risk of having AP increased significantly if the tooth had a deep carious extent. ${ }^{5}$

Permanent first molar due to its early eruption and the posterior location is most caries prone and most treated tooth in dentistry.

In the present study, mandibular molars were included because of their root morphology as they have two roots. Hence, the correlation of caries distribution with the periapical status could be done easily. The literature has shown a more frequent association of AP with mandibular molars. ${ }^{7}$

$\mathrm{AP}$ is an inflammatory process in the periapical tissues that may develop by the penetration of bacteria into the dental pulp. The relation between AP and bacteria infecting the root canal system is well established in experimental studies. ${ }^{8-9}$ An infection of the coronal pulp may spread apically, and cause necrosis of the pulpal tissues, and eventually reach the apical part of the root canal. If left untreated, bacterial elements invade the periapical area and cause local bone destruction.

In order to evaluate the prevalence of AP, complete series of periapical radiographs have been used because they provide better images for the examination of the apical periodontium. Although many studies have been carried out with panoramic radiographs, many other 
authors have chosen a complete series of periapical radiographs or the association of both..$^{10-12}$

There are different criteria for diagnosing AP radiographically (PAI), periapical, and endodontics status scale (PESS), (CBCT PAI). ${ }^{13}$ In this study, PAI proposed by Orstavik et al. was used because it is easy to reproduce and facilitates comparison to other epidemiological studies. ${ }^{14} \mathrm{PAI}$ is an index that can be operational and is reliable. ${ }^{15}$

Therefore, in present study topographic distribution of caries and radiographic analysis (using PAI) was evaluated to see the effect of anatomical site of caries, individual anatomical form of root and extent \& severity of apical periodontitis developed consequently by PAI.

\section{MATERIALS AND METHOD}

It is a retrospective single-centre observational study carried out in the department of Pedodontics and Preventive Dentistry, SGT Dental College, Gurugram. Clinical records of 250 children were selected from the departmental records, who visited the department between August 2018 and August 2019. Out of 250, 100 intraoral periapical radiographs were selected based on inclusion and exclusion criteria.

Inclusion and exclusion criteria: Deep carious, pulpally involved young permanent mandibular molars with a distinct cavity or extensive distinct cavity with visible dentin, an extensive cavity involving at least half of a tooth surface and possibly reaching the pulp [International Caries Detection and Assessment System (ICDAS Score 5, 6)] were included. Whereas the periapical radiographs which were not clearly visible, had artifacts (i.e. dark, light radiographs, apical portion of root not visible, etc) and endomolaris: mandibular molars with extra third root were excluded from the study.

An experienced single clinician with clinical experience of at least 5 years participated in the study as an examiner. The examiner was calibrated before radiographic evaluation, for such purpose and 100 periapical radiographs were examined. As part of the calibration phase, the study methodology was explained to the examiner; familiarized with the scores they should attribute to the periapical radiographic images, as well as with the established evaluation method for the study, to minimize discrepant results and provide greater reliability to the scores (figure 1).

A qualified radiographer had taken all the radiographs before the clinical examination. The radiographs were evaluated for the caries distribution pattern (mesial, distal and occlusal).

Further, the caries distribution was correlated with periapical radiolucency, using Periapical Index (PAI) proposed by Ørstavik et al, ${ }^{14}$ according to which 5 scores were attributed to the apical area of the radiographic images, as follows: 1 . normal periapical structures; 2. Small changes in the bone structure; 3. changes in the bone structure with mineral loss; 4 . periodontitis with the well-defined radiolucent area; 5 . severe periodontitis with exacerbating features. Before starting the analysis, the proposed criteria for scoring the teeth with AP were explained to the examiner: 1) choose the score that most closely represented the apical periodontium status of the tooth under analysis: 2) in case of doubt, the highest score should be given; 3) all teeth with AP should be scored 3, 4 and 5, i.e., those with chronic apical periodontitis.

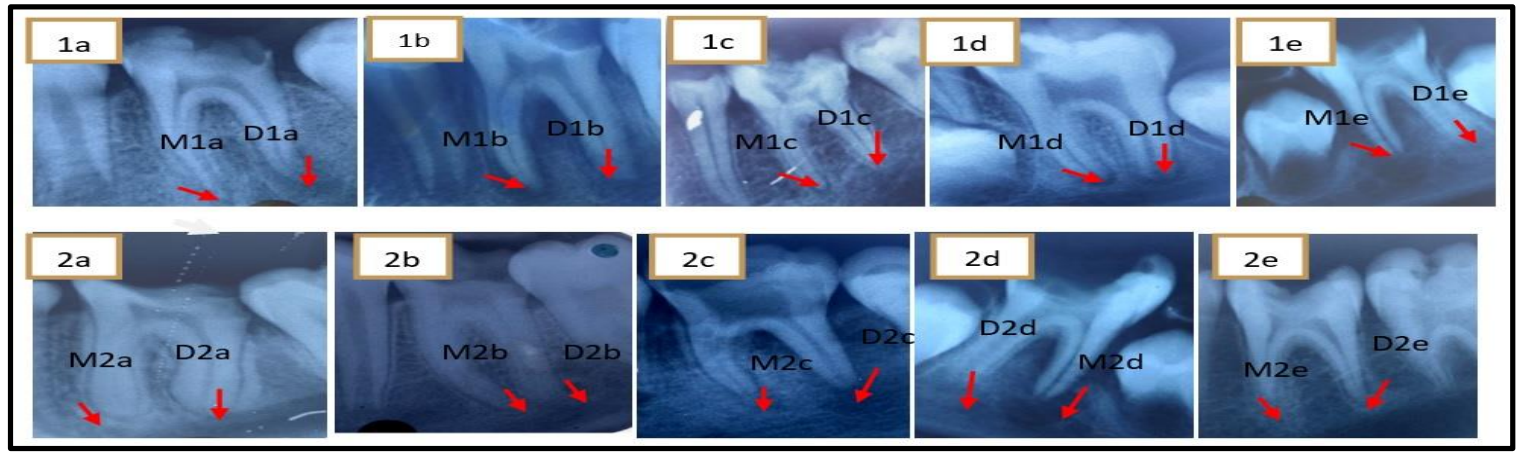

Figure 1. Intraoral periapical radiographs showing different PAI scoring-- $1 \mathrm{a}($ Mia - 2 and Dia - 4), $1 \mathrm{~b}($ Mib and Dib - 4), 1c (Mic and Dic - 2), $1 d$ (M1d and Did-3)and 1e(M1e - 4 and Die - 3), 2a (M2a and D2a-3), 2b (M2b and D2b- 2), 2c (M2C-3 and M2d-4), 2d (M2d-4 and D2d-3), and 2e (M2e and D2e-3). 


\section{RESULTS}

A total of 100 periapical radiographs representative of 8o patients, 100 young permanent teeth were examined. Both sides i.e. the right and left mandibular molars were equally affected. Of the teeth examined, most common was occlusal caries i.e. $54 \%$ followed by $32 \%$ disto-occlusal and $14 \%$ had mesio-occlusal caries (table 1).

\begin{tabular}{|c|c|c|}
\hline Parameter & Number & Percentage \\
\hline Mesial & 14 & $14 \%$ \\
\hline Distal & 32 & $32 \%$ \\
\hline Occlusal & 54 & $54 \%$ \\
\hline
\end{tabular}

Table 1. Distribution of caries on different surfaces

In case of distal root PAI-Out of 14 cases of mesioocclusal caries $4,(28.6 \%)$ cases had more than 2 score and $10(71.4 \%)$ had less than 2 PAI score, whereas in cases of disto-occlusal caries, out of 32 cases $13(40.6 \%)$ had more than 2 score and $19(59.4 \%)$ cases had less than 2 score, which is statistically insignificant (pvalue-0.576 and 0.591) respectively (figure 2).

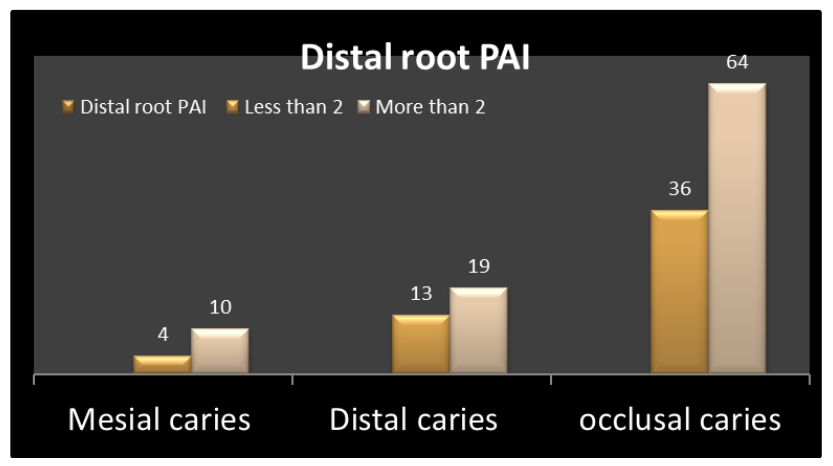

Figure 2. Relationship of mesial, distal and occlusal caries with distal root (PAI)

In case of mesial root, PAI - out of 14 cases of mesioocclusal caries, 7 (50\%) cases had more than 2 score and $7(50 \%)$ cases had less than 2 PAI score, in distoocclusal caries out of 32 cases, 20(60.5\%) cases had more than 2 score and $12(37.5 \%)$ cases had less than 2 score and the results were insignificant with p-value0.576 and 0.765 respectively (figure 3 ).

On comparing more than 2 PAI score on the mesial and distal root, the mesial root had more PAI score in case of mesio-occlusal caries whereas the distal root shows

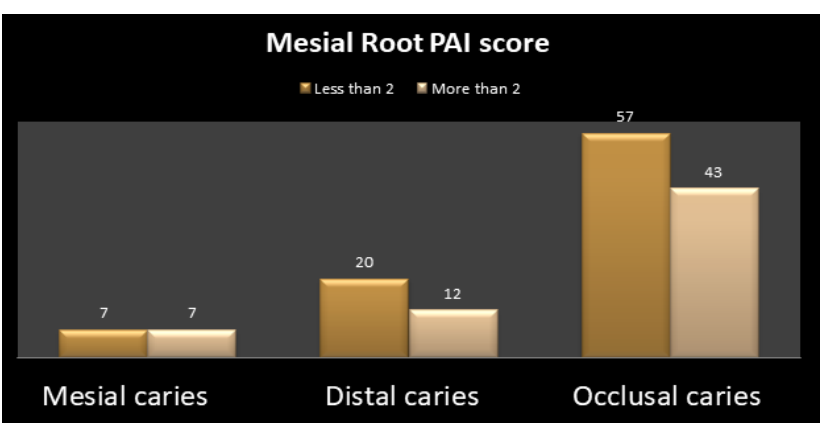

Figure 3. Relationship of mesial, distal, occlusal caries with mesial root PAI

more PAI score in case of disto-occlusal caries (Figure 4).

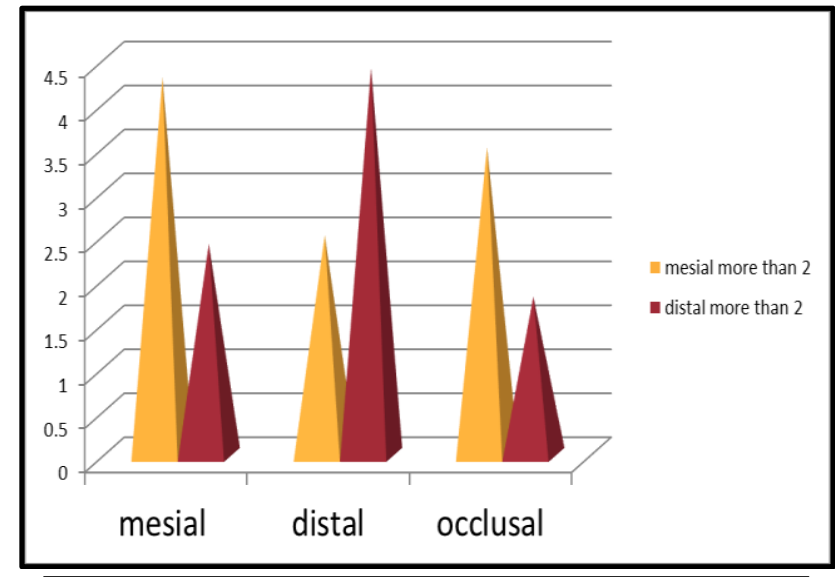

Figure 4. Distribution of caries and its relation to more than 2 PAI score in mesial and distal root

\section{DISCUSSION}

The main concern of this paper was to evaluate the distribution of caries in young permanent mandibular molars and its relation to the extent and severity of apical periodontitis.

For the diagnosis of the apical periodontitis and its relation to caries distribution, intraoral periapical radiographs were used. Some investigators suggested that in epidemiologic studies the use of a panoramic radiograph of dental health was acceptable and the validity and reliability of panoramic radiography for evaluating the AP is sustainable. ${ }^{16}$ In $2 \mathrm{D}$ dental radiographs, there is a lack of information in the buccolingual plane and superimposition of structures, CBCT images are more accurate for detection of AP. But the $\mathrm{CBCT}$ examination generates more radiation 
exposure to the patient. ${ }^{17}$ However, others found that periapical radiographs are superior to panoramic radiographs in the detection of periapical lesions because panoramic radiography may under-estimate lesions that occurred when used. The radiographs of high quality were included to minimize radiographic errors. $^{18}$

The diagnosis of apical periodontitis is useful because distal roots development completion takes a longer time. According to results, preventive informative education can be planned.

There are various criteria for diagnosing AP radiographically, and they vary among amongst the studies. In the present study, the Periapical Index (PAI) proposed by Ørstavik et al. was used because it facilitates the comparison to other epidemiological studies and is easily reproducible.

The increased incidence of caries on the occlusal and proximal surfaces can be correlated to the morphology of these sites, which is more retentive of food particles, relatively less protected from mechanical cleaning by the tongue, cheeks, and tooth brushing, and is not fully exposed to the flushing action of saliva. ${ }^{18-19}$

Distal root PAI is more than that of mesial root-because the distal canal is evidently straight and wider, and the apex closure is after mesial root apex. Therefore caries progresses more rapidly in the distal canal.

\section{Limitations:}

-Intraoral periapical radiographs were used in the study, which were not clear as high resolution digital radiographs.

-In present study, 2D dental radiographs were used, which lack the information in the buccolingual plane. Further high-quality prospective study with a greater number of samples is required to determine the relation between the caries and the apical periodontitis.

\section{CONCLUSION}

In the present study, it was observed that occlusal surfaces of permanent mandibular molar are predominantly involved followed by distal and mesial surface; Distal root PAI is more than that of the mesial root, which is independent of the topographic distribution of caries.

\section{REFERENCES}

1. Hassan A, Khan JA, Ali SA. Caries Susceptibility of Proximal Surfaces in Permanent First Molars: A Cross Sectional Survey. JIIMC 2019;14(1):38-42.

2. Nazir MA, Bakhurji E, Gaffar BO, Al-Ansari A, AlKhalifa KS. First Permanent Molar Caries and its Association with Carious Lesions in Other Permanent Teeth. J Clin Diagn Res. 2019;13(1): ZC36-ZC39. https://doi.org/10.786o/JCDR/2019/38167.12509

3. Srinath S, Somasundaram J. Dimensions of Pulp Chamber and Furcal Dentin in Mandibular First Molar in South Indian Population-A Radiographic Study. J Pharm Sci Res. 2017;9(7):1106.

4. Abuaffan AH, Hayder S, Hussen AA, Ibrahim TA. Prevalence of dental caries of the first permanent molars among 6-14 years old Sudanese children. Indian J Dent Educ. 2018;11(1):13-6.

5. Kirkevang LL, Væth M, Hörsted-Bindslev P, Bahrami G, Wenzel A. Risk factors for developing apical periodontitis in a general population. Int Endod J. 2007;40(4):290-9.

6. Eriksen HM, Kirkevang LL, Petersson K. Endodontic epidemiology and treatment outcome: general considerations. Endodontic Topics 2002;2(1): 1-9. https://doi.org/10.1034/j.1601-1546.2002.20101.X

7. Buckley M, Spangberg LS. The prevalence and technical quality of endodontic treatment in an American subpopulation. Oral Surgery, Oral Medicine, Oral Pathology, Oral Radiology, and Endodontology. 1995;79(1):92-100.

8. Möller ÅJ, Fabricius L, Dahlen G, Ohman AE, Heyden GU. Influence on periapical tissues of indigenous oral bacteria and necrotic pulp tissue in monkeys. Eur J Oral Sci. 1981;89(6):475-84.

9. Kakehashi S, Stanley HR, Fitzgerald RJ. The effects of surgical exposures of dental pulps in germ-free and conventional laboratory rats. Oral Surg Oral Med Oral Pathol. 1965;20(3):340-9.

10. Maslamani M, Behbahani J, Mitra AK. Radiographic evaluation and predictors of periapical lesions in patients with root-filled and non root-filled teeth in Kuwait. Indian J Dent Sci. 2017;9(4):237.

11. Marques MD, Moreira B, Eriksen HM. Prevalence of apical periodontitis and results of endodontic treatment in an adult, Portuguese population. International Endodontic Journal 1998;31(3):161-5. 12. Loftus JJ, Keating AP, McCartan BE. Periapical status 
and quality of endodontic treatment in an adult Irish population. International endodontic journal. 2005;38(2):81-6.

13. Venskutonis T. Periapical tissue evaluation: analysis of existing indexes and application of Periapical and Endodontic Status Scale (PESS) in clinical practice. G Ital Endod. 2016;30(1):14-21.

14. Orstavik D, Kerekes K, Eriksen HM. The periapical index: a scoring system for radiographic assessment of apical periodontitis. Dent Traumatol. 1986;2(1):20-34. 15. Terças AG, Oliveira AE, Lopes FF, Maia Filho EM. Radiographic study of the prevalence of apical periodontitis and endodontic tratment in the adult population of São Luís, MA, Brazil. J Appl Oral Sci. 2006;14(3):183-7.

16. Fernandes LM, Ordinola-Zapata R, Húngaro MAD, Capelozza ALA. Prevalence of apical periodontitis detected in cone beam CT images of a Brazilian subpopulation. Dentomaxillofac Radiol. 2013; 42(1): 80179163.

17. Nair PN. Pathogenesis of apical periodontitis and the causes of endodontic failures. Critical Reviews in Oral Biology \& Medicine. 2004;15(6):348-81.

18. Dawson VS, Petersson K, Wolf E, Åkerman S. Periapical status of root-filled teeth restored with composite, amalgam, or full crown restorations: a cross-sectional study of a Swedish adult population. J Endod. 2016;42(9):1326-33.

19. Chala S, Abouqal R, Abdallaoui F. Prevalence of apical periodontitis and factors associated with the periradicular status. Acta Odontol Scand. 2011;69(6):355-9.

\section{AUTHOR AFFILIATIONS: (*Corresponding Author)}

1. Post Graduate Student (https://orcid.org/oooo-0002-6501-1486)

2. Professor (https://orcid.org/oooo-0001-5931-0693)

3. Senior Lecturer [(https://orcid.org/oooo-0oo3-1000-3486, Dr. Sakshi Joshi), (https://orcid.org/oooo-0002-4166-3965, Dr. Shikha Dogra)]

4. Professor \& Head of the Department (https://orcid.org/oooo-0002-7848-942X)

5. Reader (https://orcid.org/oooo-0003-4429-6387)

Department of Pediatric \& Preventive Dentistry, Faculty of Dental Sciences, SGT University, Gurugram 